\title{
PHP-Nuke: software de código abierto en la gestión de contenidos web
}

\section{Introducción}

ANTE EL GRAN INCREMENTO de información en el web, cada vez son más las empresas o instituciones que optan por implementar un sistema de gestión de contenidos, tanto en sus intranets como en la información que presentan a sus clientes o usuarios.

A pesar de que las grandes empresas como Vignette o Microsoft ofrecen productos comerciales (Vignette Content Management o Microsoft Content Server respectivamente), en un entorno de restricciones presupuestarias, son muchos los que optan por escoger programas que se ofrecen en modalidades "freeware" a través de la Red.

http://www.vignette.com/contentm anagement/

http://www.microsoft.com/cmserver/

\section{Por Josep Manuel Rodríguez i Gairín}

En este trabajo presentamos una descripción de los mismos, sus principales características y módulos, así como algunas reflexiones sobre las ventajas e inconvenientes en su elección.

\section{¿Qué es un CMS?}

Las siglas CMS (del término inglés Content Managenent Systems) identifican un conjunto de programas informáticos destinados a gestionar la presentación de los contenidos de una sede web. Estos productos requieren un sistema de gestión de bases de datos vía web, un servidor web y un lenguaje de programación de las páginas.

La mayor parte de los CMS que se distribuyen por internet de manera gratuita se basan en la tríada Apache, MySQL y PHP.

-El servidor web Apache es uno de los más extendidos. Aun- que generalmente se ha asociado a entornos Linux o Unix, se distribuyen versiones para Windows $N T / 2000 / X P$. En cualquiera de los casos su instalación es muy simple y rápida, pudiendo incluso instalarse en un Pc local para poder hacer pruebas preliminares.

http://httpd.apache.org/

- MySQL es a su vez uno de los sistemas de gestión de bases de datos relacionales más potentes y robustos que se distribuyen de manera gratuita por internet. Dejando aparte conocidos sistemas comerciales como Oracle o Microsoft $S Q L$ Server, MySQL tiene entre sus competidores principales PostgreSQL, un sistema desarrollado por la Universidad de California que también se distribuye como sistema abierto.

http://www.mysql.com

http://www.postgresql.org/ 


\section{baratz}

En primera línea en

Sistemas de Información y Gestión del Conocimiento

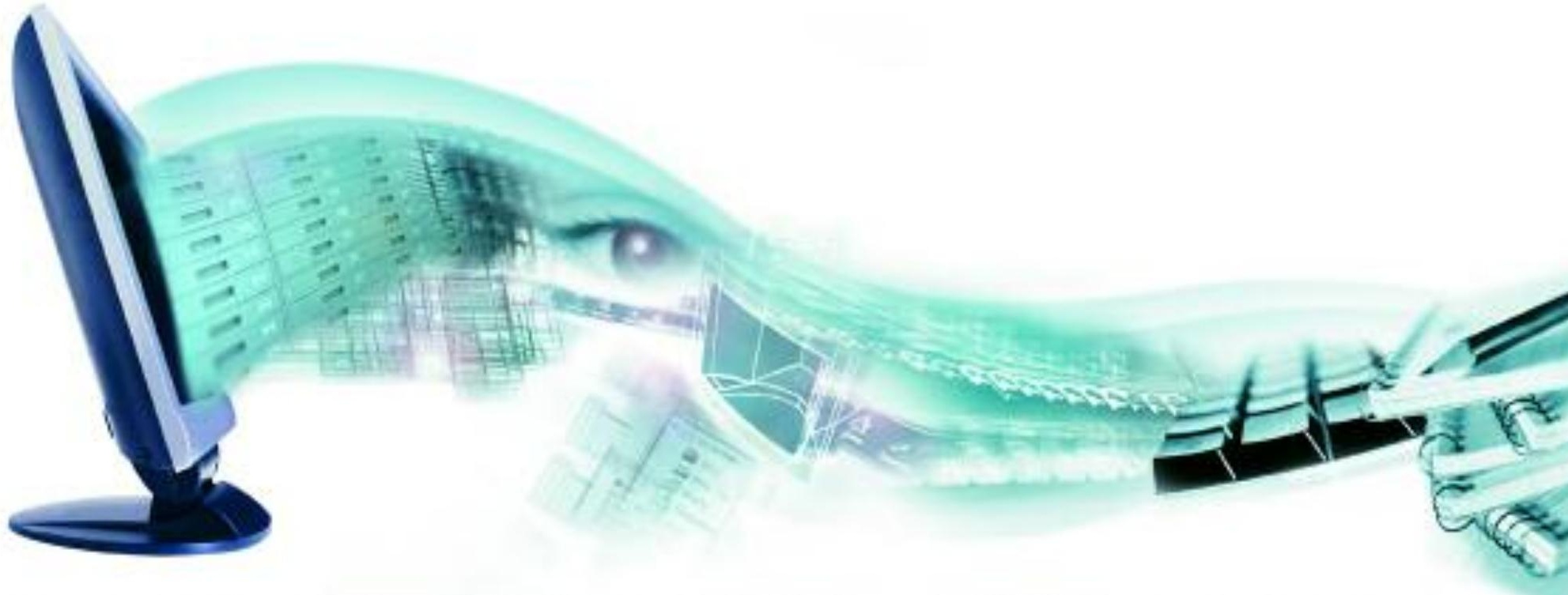

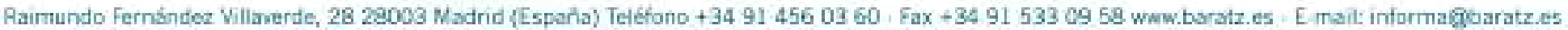

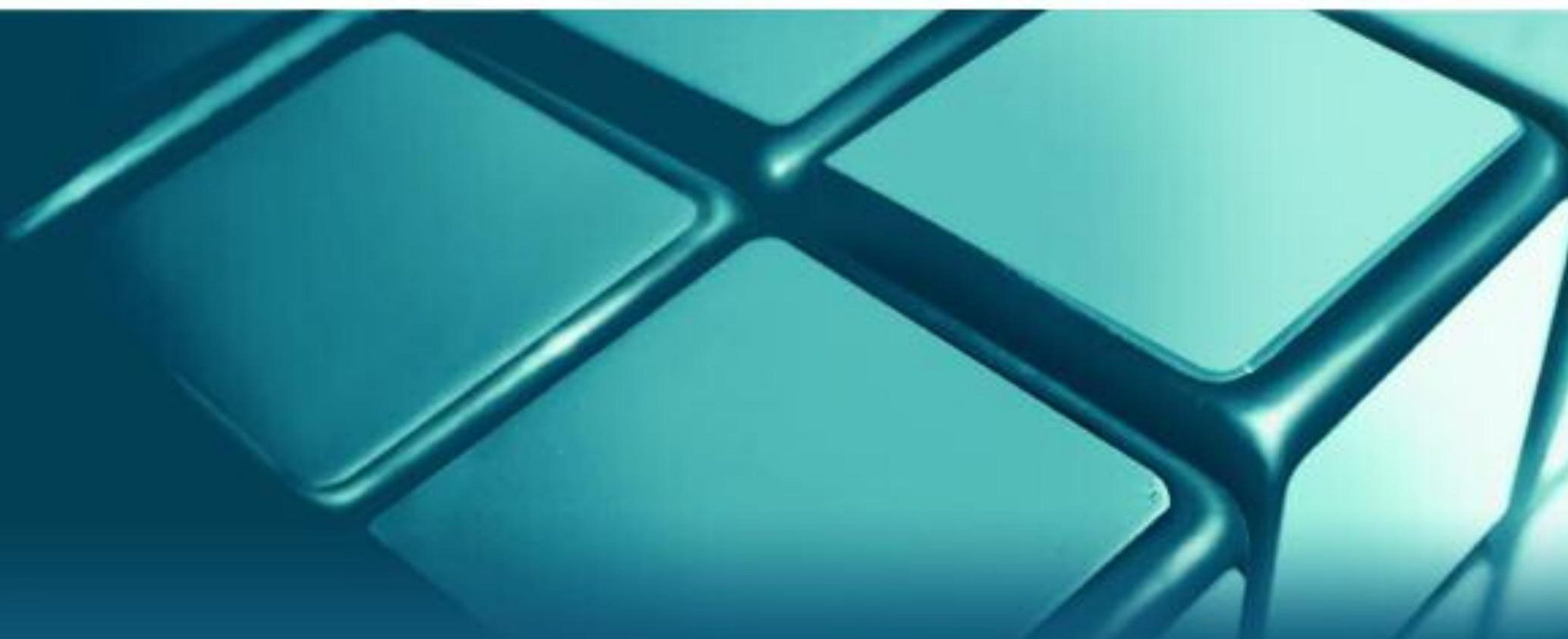

- Soluciones para bibliotecas:

Absys, absys

- Gestión documental y del conocimiento:

BKM, Baratz Windows, BRSCGI

- Soluciones para archivos: Albalá

- Servicios de Catalogación Retrospectiva

- Edición en CD-Rom

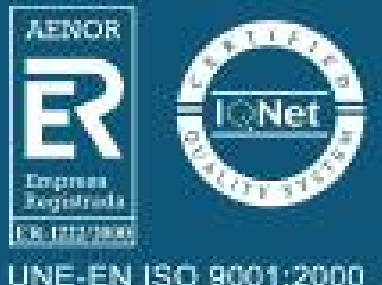


Todos ellos emplean SQL, un estándar desarrollado por $I B M$ en los años 70 para la gestión de bases de datos relacionales que, a efectos prácticos, nos permite la importación/exportación de los datos con cualquier producto que lo utilice. Desde el punto de vista documental, a pesar de ser MySQL un sistema de gestión relacional, incluye sistemas de recuperación de texto completo basados en relevancia que, aunque rudimentarios, son una de las líneas de trabajo de sus creadores.

\section{«Desde este punto de vista, PHP-Nuke es uno de los más antiguos y cuenta con una amplia expe- riencia, muchos usuarios y una gran cantidad de módulos adicionales»}

Al igual que en el caso de Apache, existen versiones para múltiples sistemas operativos incluidos Linux y Windows y su instalación tampoco presenta ninguna complejidad.

- PHP son siglas que, aunque originalmente significaban Personal Home Page, mejor debemos asociar a Hypertext Preprocessor ya que se trata de un lenguaje de programación que se ejecuta en el servidor antes de enviar la información al navegador. Esto permite realizar acciones como consultar bases de datos o dar formato a los resultados. $P H P$ es junto a $A S P$ (Active Server Pages) los dos lenguajes de programación de este tipo más extendidos en internet. PHP también puede instalarse tanto en Linux como en Windows $\mathrm{u}$ otros sistemas.

http://www.php.net

Los CMS que aquí comentamos son en realidad un conjunto de programas (scripts) escritos en algunos de estos lenguajes, general- mente $P H P$, cuya función es gestionar los contenidos de la sede web.

\section{¿Qué CMS debo escoger?}

En internet podemos encontrar gran cantidad de gestores de contenidos. Uno de los repositorios mas empleados para localizarlos es HotScripts (buscando bajo Home :: PHP :: Scripts and Programs :: Content Management). Un recurso más útil es OpensourceCMS, una sede web en la que podemos probar directamente los productos sin necesidad de ninguna instalación por nuestra parte. OpensourceCMS tiene preinstalados los sistemas en su servidor y ofrece la posibilidad de conectarse como usuario o como administrador modificando en ellos todo lo que queramos con total libertad ya que cada dos horas un programa les permite recuperar su estado original.

http://www.hotscripts.com http://test.opensourcecms.com/

Es difícil establecer un criterio para escoger uno u otro, si bien algunos presentan características particulares, la mayoría ofrecen un núcleo de prestaciones similares que describiremos en el siguiente apartado. Tal vez uno de los elementos que pueden ayudarnos en la elección es la cantidad de usuarios que lo están empleando, en parte, porque a mayor número de personas que lo utilizan mayor posibilidad de encontrar soluciones a problemas en los foros y de encontrar otros desarrollos (módulos) para ese entorno concreto. Desde este punto de vista, PHP-Nuke es uno de los más antiguos y cuenta con una amplia experiencia, muchos usuarios y una gran cantidad de módulos adicionales. Mencionar también PostNuke y Typo3, este último algo más complejo de configurar pero con una gran potencialidad en la gestión de niveles de seguridad, aspecto importante en el manejo de intranets corporativas.

http://www.phpnuke.org/

http://www.postnuke.com/ http://typo3.com/

\section{¿Qué opciones ofrecen los CMS?}

La mayoría se estructuran en forma de módulos que ofrecen funcionalidades específicas. Con la instalación se suministran unos módulos básicos, pero en internet es posible encontrar otros muchos, elaborados por los usuarios, y distribuidos libremente a través de repositorios y foros.

Los $C M S$ presentan tres características genéricas:

-Personalización: la pantalla principal de acceso se divide en tres o cuatro zonas. En la zona superior se coloca la cabecera, un es-

Algunos ejemplos de revistas publicadas con
PHP-Nuke o similares:
— Revista del Vallès
http://revistadelvalles.com/
—La revista @ pangea.
http://revista.pangea.org/
—Senda. La revista de los senior.
http://www.sendasenior.com/
—Revista agoraNet.
http://cibercafe.esplugues.net/phpnuke/index.php
—MidWest technology journal.
http://www.pcjournal.com/


pacio central se reserva para los contenidos propiamente dichos $\mathrm{y}$ sendas secciones laterales para menús y opciones agrupadas en módulos.

Desde una pantalla de administración, muy sencilla de manejar, es posible cambiar los logos, las combinaciones de colores (controladas por temas u hojas de estilo), el idioma por defecto y las posiciones y contenidos de los diferentes módulos que se presenten.

Un sistema de identificación permite que los usuarios, una vez autentificados, puedan personalizar distintas opciones, desde el idioma a los contenidos específicos que aparecerán en su página personal. Existen tres niveles básicos de acceso: anónimo, usuario autentificado y administrador. Cada uno de ellos podrá visualizar o activar distintos módulos.

-Comunicación: una característica importante de todos estos sistemas es la posibilidad de comunicación con y entre los usuarios; desde enviar mensajes personalizados a otros usuarios o listas de distribución hasta participar en foros o charlas en directo. Por supuesto, todos ellos incorporan sistemas de retroalimentación, informando a los administradores de problemas o sugerencias y sistemas de valoración de los contenidos por medio de votaciones electrónicas.

-Recuperación de información: los distintos contenidos que se van ofreciendo se almacenan en tablas dentro de la base de datos. Por regla general, en cada pantalla o módulo se van presentando los últimos contenidos publicados, pero un sistema de desplazamiento o un motor de consulta permite la localización y recuperación de cualquier información almacenada.

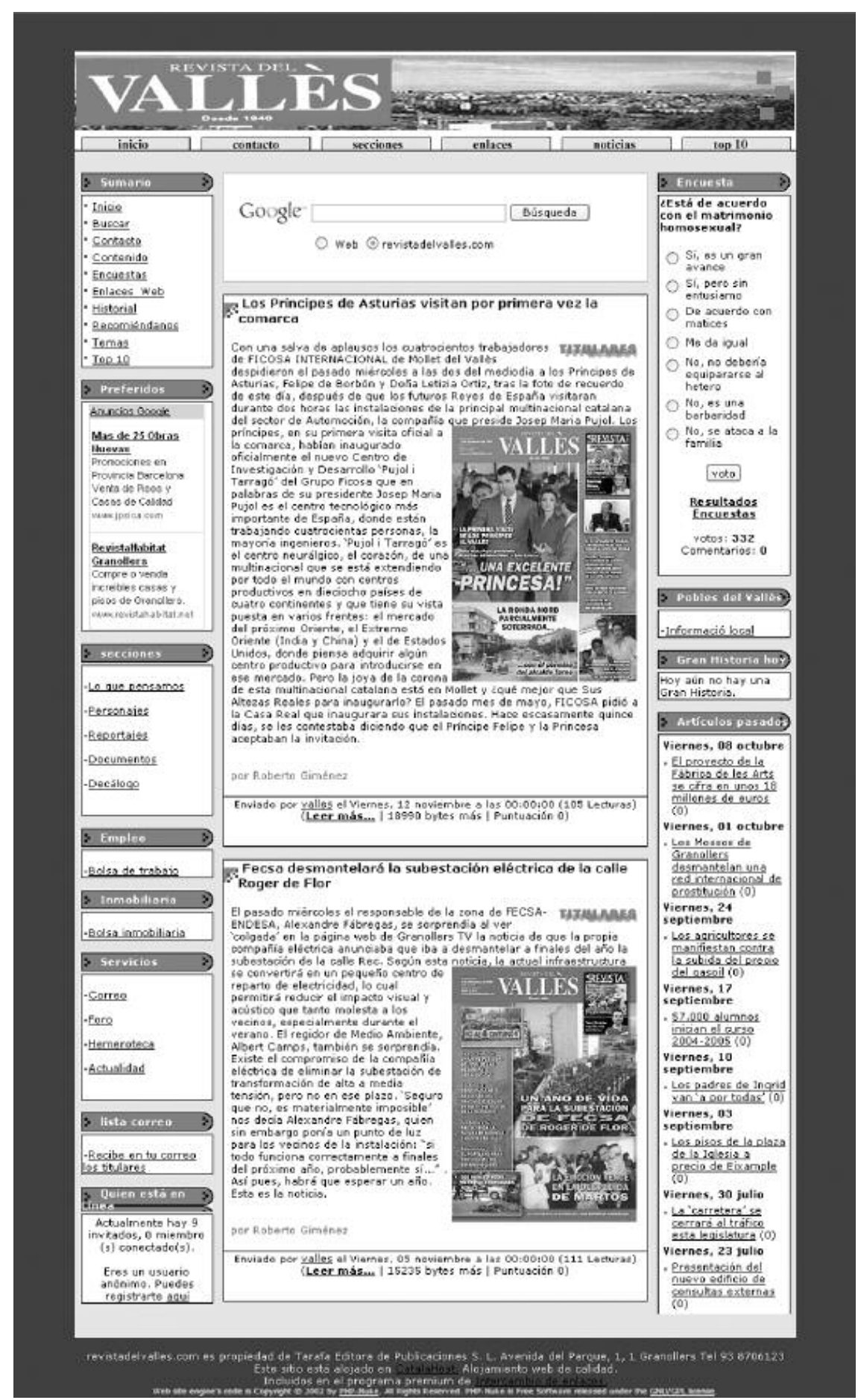

Figura 1. La revista del Vallés

\section{¿Cuáles son los principales módulos de los CMS?}

Habitualmente son los siguientes:

1. Noticias: permite al administrador de los contenidos ir introduciéndolas para presentarlas a los usuarios por orden cronológico descendente. Para cada una se introduce un texto breve, el texto completo de la misma así como una asignación a diferentes categorías. El administrador puede permitir a los usuarios añadir comentarios o votarlas. El usuario no puede introducir noticias directamente pero sí puede enviarlas al administrador para su valoración y posterior inclusión (ver ejemplos de revistas-e que utilizan este módulo en la tabla 1).

Ofrece una gran versatilidad y no debe asociarse únicamente al 
concepto noticias. Por ejemplo, puede ser usado perfectamente para la gestión de una revista electrónica, hablando de artículos agrupados en secciones y permitiendo a los lectores la recuperación de artículos anteriores así como adjuntar comentarios o votarlos. Un buen ejemplo de gestión integral de una revista con PHP-Nuke lo podemos encontrar en: Revista del Vallés (figura 1).

\section{http://revistadelvalles.com/}

Asociado a este módulo, muchos $C M S$ incorporan tecnologías capaces de procesar RSS, un formato $X M L$ para la sindicación de contenidos, capaz de ofrecer a los usuarios noticias de terceros. $\mathrm{Mu}$ chas agencias de información y diarios están ya empleando estos formatos para la distribución de las últimos titulares, como por ejemplo El país.

http://www.elpais.es/rss.html

2. Forum: es el núcleo principal de comunicación en este tipo de productos. Los distintos foros pueden configurarse de múltiples

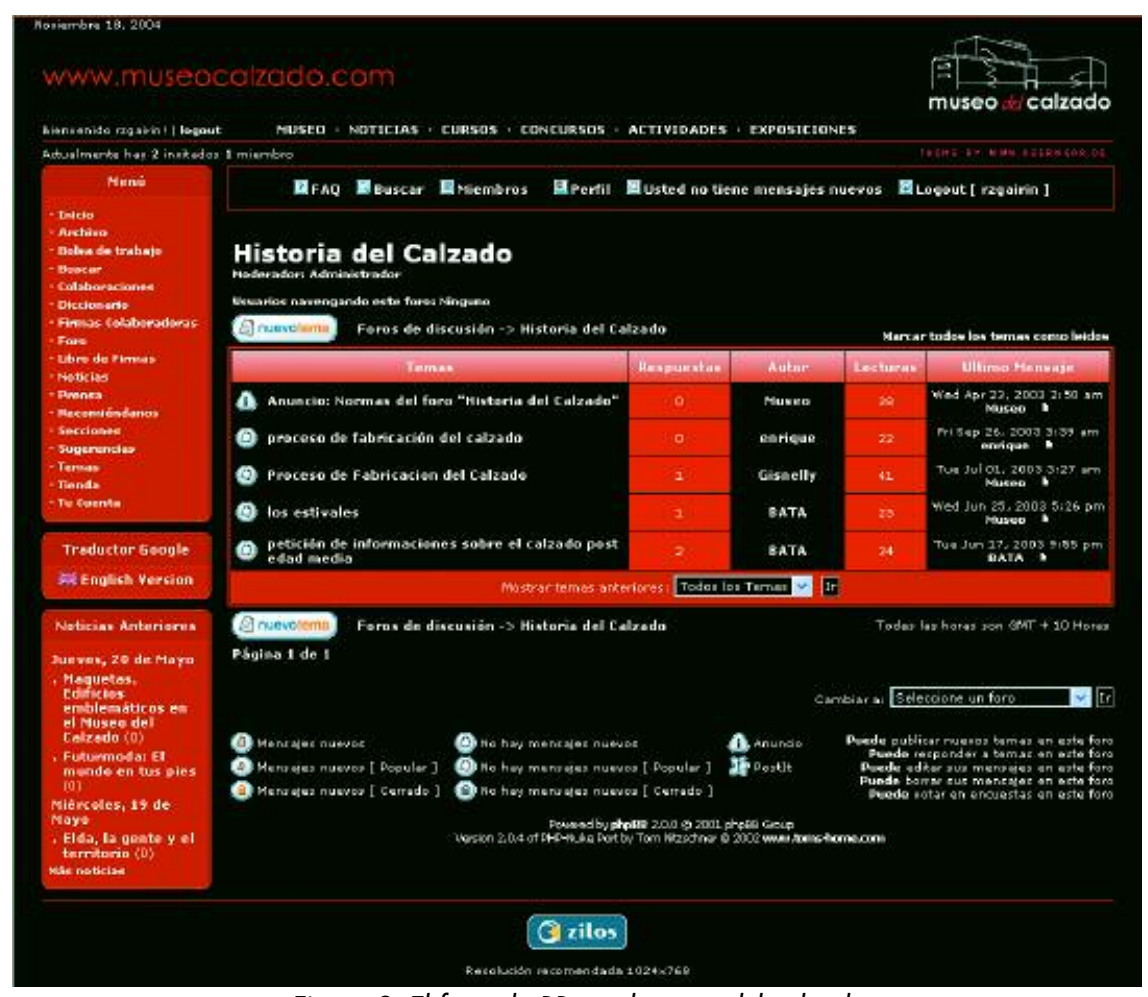

Figura 3. El foro phpBB en el museo del calzado

maneras. Un foro abierto permite la comunicación global entre todos los usuarios, pero también puede plantearse la utilización de este módulo para enviar preguntas a un servicio de referencia y ofrecer las respuestas públicamente. $\mathrm{PHP}-\mathrm{Nu}$ -

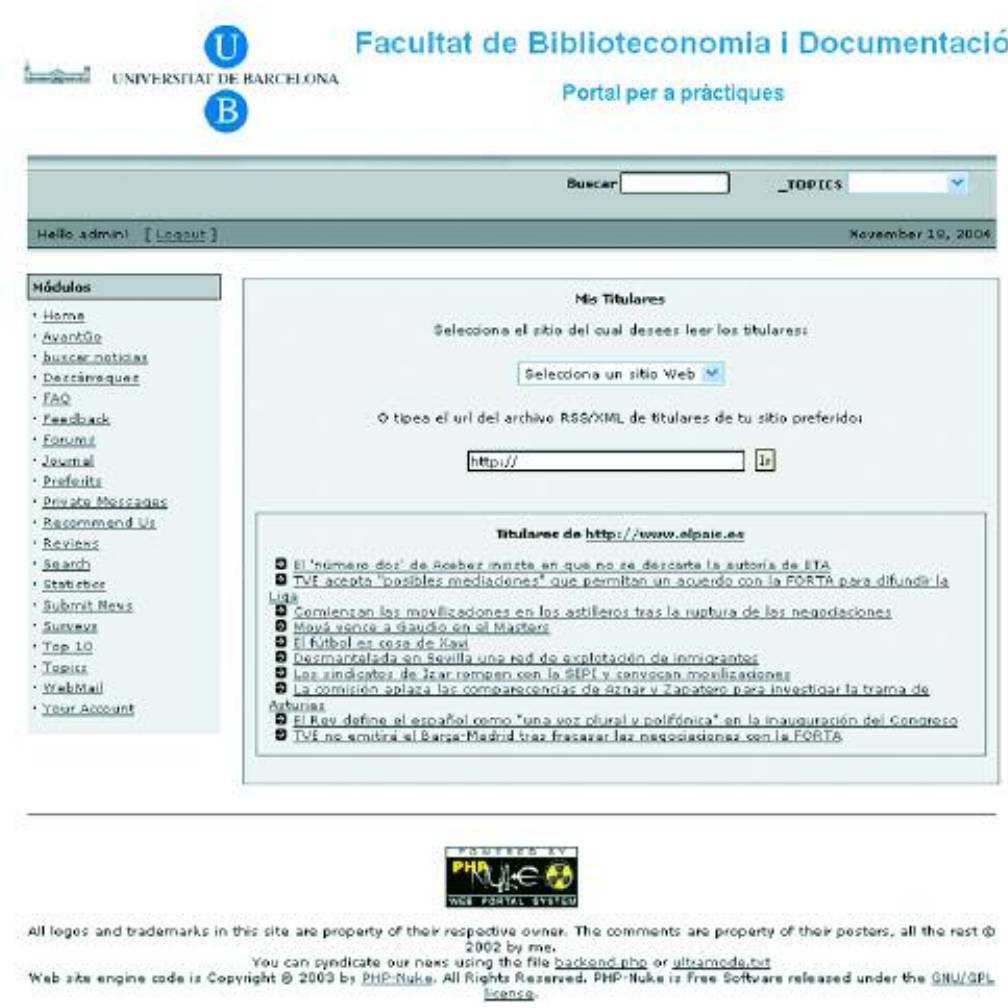

Figura 2. Un ejemplo de titulares integrados en PHP-Nuke ke no incorpora directamente un módulo de estas características, pero podemos instalar el programa phpBB que se integra perfectamente en la mayoría de $C M S$ de código libre.

\section{http://www.phpbb.com/}

3. Módulos de enlaces y de descargas: el primero actúa a modo de "bookmark" o libro de favoritos pero compartido por todos los usuarios del sistema. Es una funcionalidad que muchas bibliotecas y centros de documentación siempre han deseado implementar en sus sedes web. Los enlaces se organizan por categorías y a su vez puede retroalimentarse con las aportaciones de todos los participantes, supervisadas por el administrador quien siempre ha de verificar el correcto funcionamiento del enlace y activar su visualización.

El módulo de descargas funciona de manera similar al de enlaces. Suele emplearse para organizar y facilitar la descarga de programas informáticos, pero también se puede utilizar para la distribución de documentos electrónicos. Un ejemplo de esto último pode- 


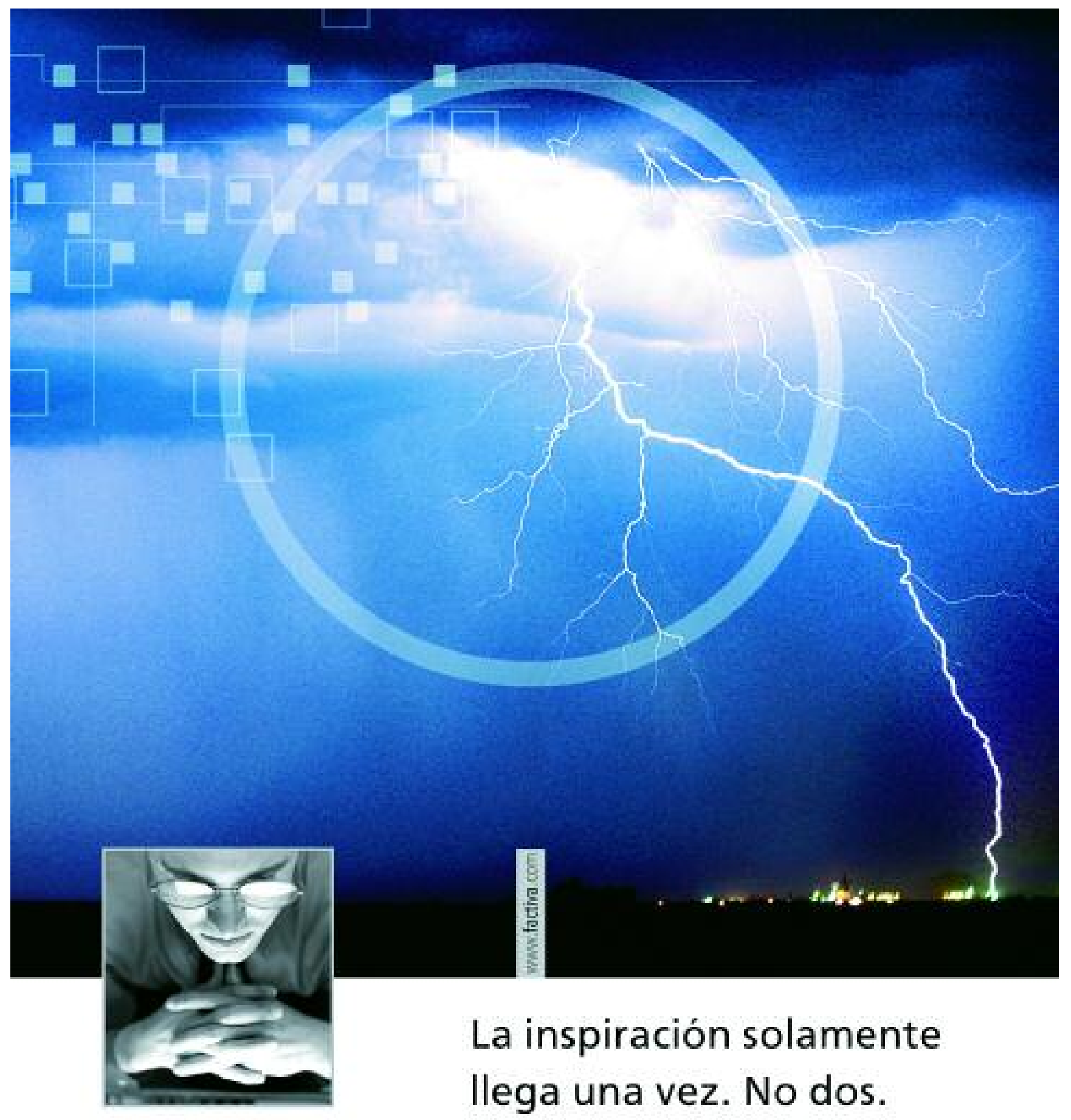

Para toda decisión importante no se tiene normalmente una segunda oportunidad. Cuando llega el momento de la verdad. usted necesita acceso inmodiato a una información precisa y fiable.

Para responder eficazmente, Faxtiva le da acceso a informacion profesional y económica, bcal e internscional, para inspirarle en sus mejores decisiones. Cuando usted lo necesita, comousted lo necesita.

Para más información vishte www.factivacom/es/inspiration 
mos verlo en la editorial argentina ArtNovela desde cuyo portal, desarrollado en PHP-Nuke, es posible obtener libros enteros mediante el módulo de descargas (figura 4).

http://www.artnovela.com.ar

4. Preguntas frecuentes: permite presentar las preguntas más comunes formuladas a los responsables de la sede web y sus respuestas a fin de aligerar la sobrecarga de los centros de asistencia al usuario. Puede ser de gran utilidad tanto en empresas privadas (en especial de servicios) como en bibliotecas o centros de documentación.
5. Módulo de diarios personales: conocidos también como weblogs, son herramientas de comunicación que han cobrado especial relevancia en los últimos años. Los usuarios de un CMS como PHPNuke pueden activar su diario personal y compartirlo con el resto de la comunidad, incluso hacerlo público a los usuarios no registrados.

Existen muchos otros módulos específicos y utilidades asociadas a los $C M S$. En nuestro entorno destacaremos phpmylibrary, un sistema integrado de gestión de bibliotecas entre cuyos usuarios encontramos

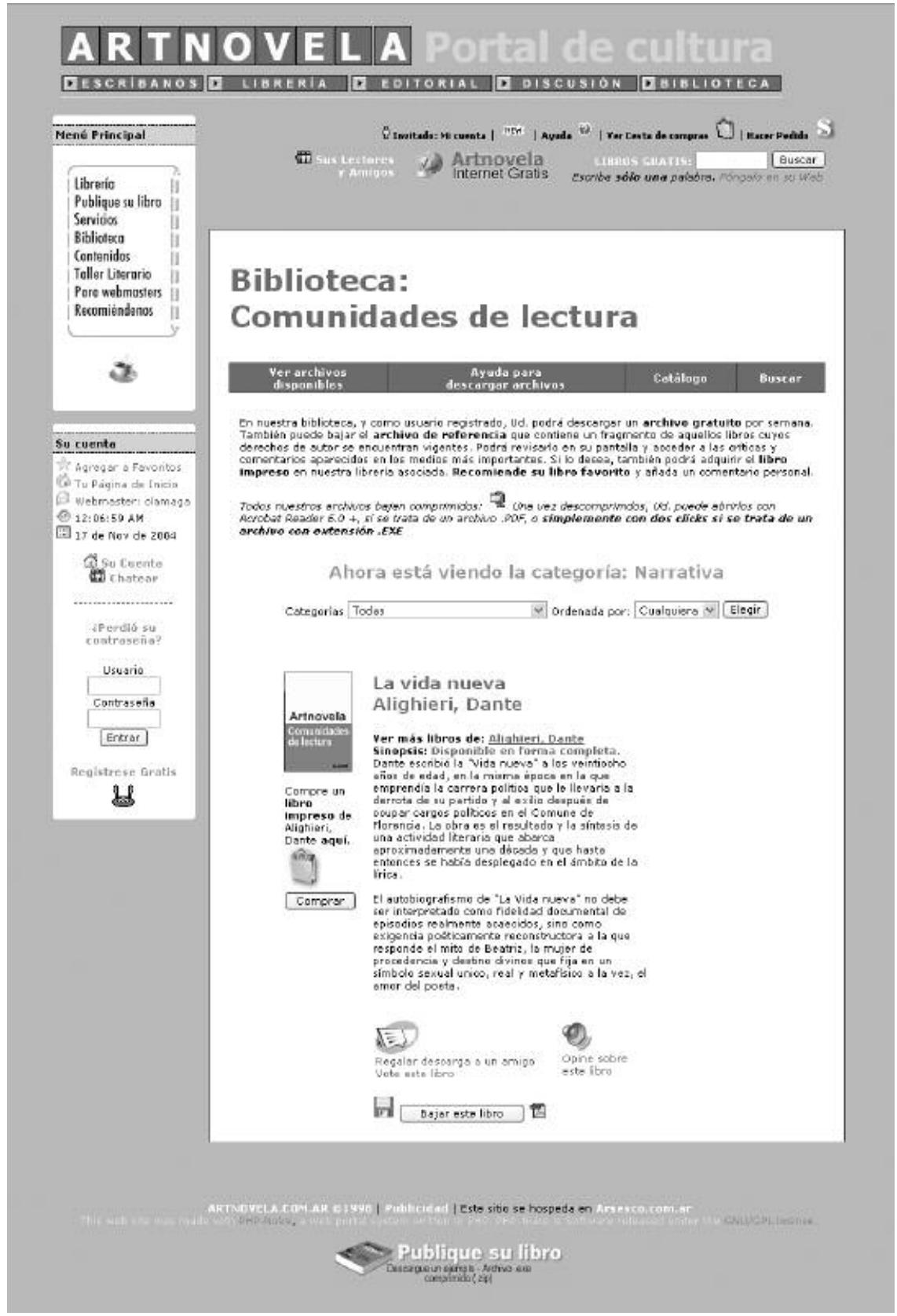

Figura 4. Modulo de descargas empleado para la distribución de libros. Editorial ArtNovela el Laboratorio de Astrofísica Espacial y Física Fundamental, así como otras bibliotecas internacionales.

http://www.phpmylibrary.org/

http://www.laeff.esa.es/modules.ph $p ?$ op $=$ modload \& name $=P h p M y L i$ brary\&file $=$ index

\section{«La duda se plantea en la decisión de op- tar por un programa comercial o de códi- go libre y gratuito»}

Desde el punto de vista de la administración de la sede web, remarcar también el sistema de registro de estadísticas que permite: conocer los accesos, usuarios, utilización del sistema y otros muchos datos útiles para tomar decisiones en la gestión de la información ofrecida.

\section{¿Comercial o de código libre?}

Aunque posiblemente no exista ninguna duda de que este tipo de sistemas son la mejor herramienta para la gestión de los contenidos de una sede web, la duda se plantea en la decisión de optar por un programa comercial o de código libre y gratuito.

Si bien el aspecto económico suele ser fundamental, es importante también valorar las posibilidades de soporte técnico y continuidad del producto. La instalación y configuración de cualquiera de ellos es relativamente simple y asistida pero no se puede negar que se requieren unos ciertos conocimientos técnicos, en especial ante la aparición de problemas. Los defensores de los productos basados en código libre abogan por la existencia de múltiples foros en donde encontrar respuestas, sin embargo dichas soluciones no siempre son sencillas de encontrar y mucho menos de una manera rápida. 


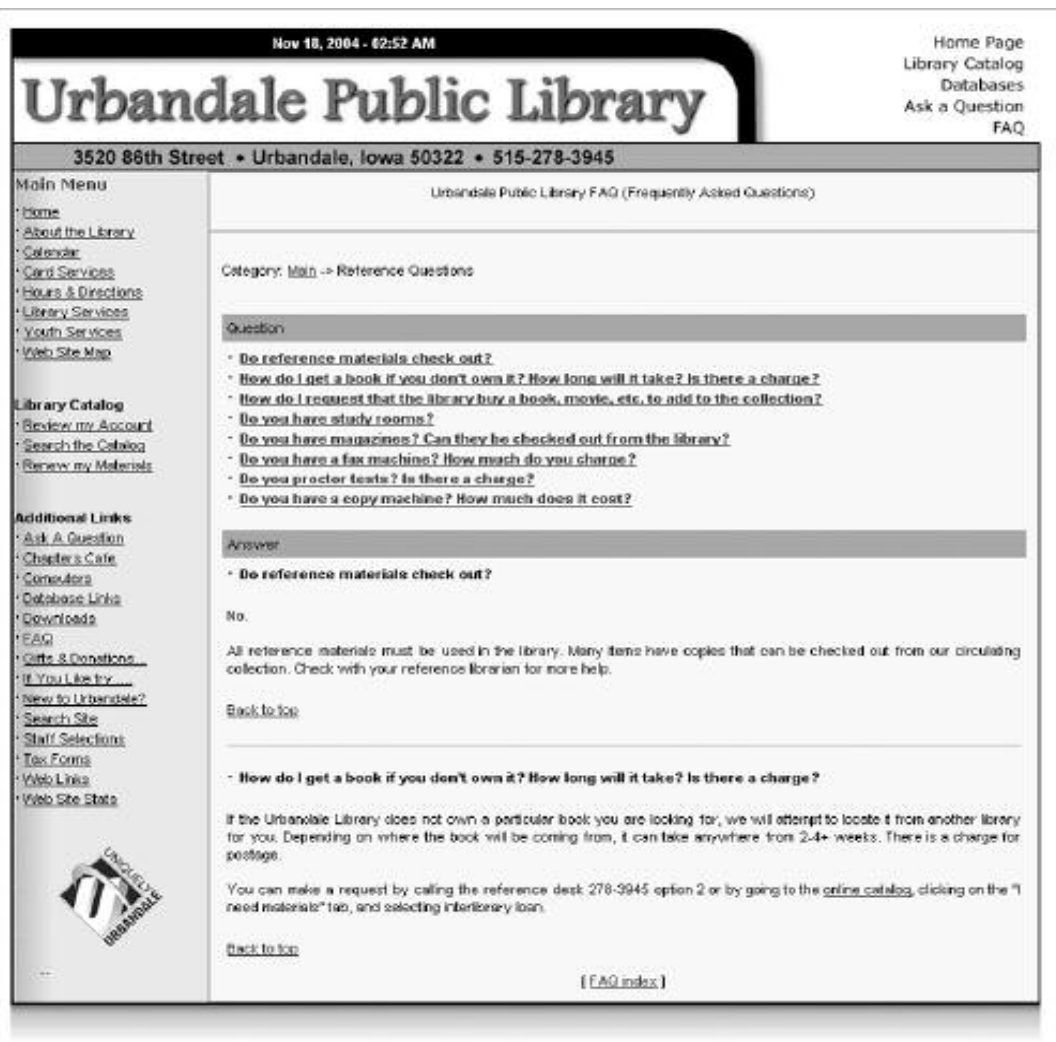

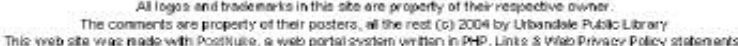

Figura 5. Módulo de preguntas frecuentes en la biblioteca pública de Urbandale

Una opción intermedia se ofrece a partir de empresas de servicios que, a un coste relativamente asequible, asesoran y pueden facilitar la instalación y configuración de estos programas así como un soporte técnico continuado.

\section{¿Algunos ejemplos?}

La mejor manera de valorar este tipo de productos es a partir de casos reales disponibles por internet. La instalación de PHP-Nuke recomienda dejar los créditos del sistema en la página principal y, aunque muchos centros los suprimen, una primera posibilidad par encontrar sedes que los usan es utilizar un buscador como Google.

A continuación listamos algunos ejemplos asociados a distintos tipos de instituciones, muchos de ellos ya comentados a lo largo de la exposición:

- Ministerio de Educación y Ciencia. Observatorio tecnológico http://observatorio.cnice.mecd.es/i ndex.php
-Ayuntamiento de Valladolid. http://www.ava.es/inicio.php

-Ayuntamiento de Peñafiel. http://www.ayto-penafiel.es

-Amposta Xarxa Ciutadana. http://amposta.org/index.php

—Lusodoc Lda (Portugal). http://www.lusodoc.pt/

\section{-Editorial Artnovela.}

http://www.artnovela.com.ar

-ClubEscritores.

http://www.clubcultura.com/ literapolis/index.php

-Museo del Calzado. http://www.museocalzado.com/ index.php

-CGT de Catalunya. http://www.cgt.es/cgtcatalunya/ index.php

- Colegio oficial de Ingenieros Industriales de Albacete. http://www.coiiab.com/

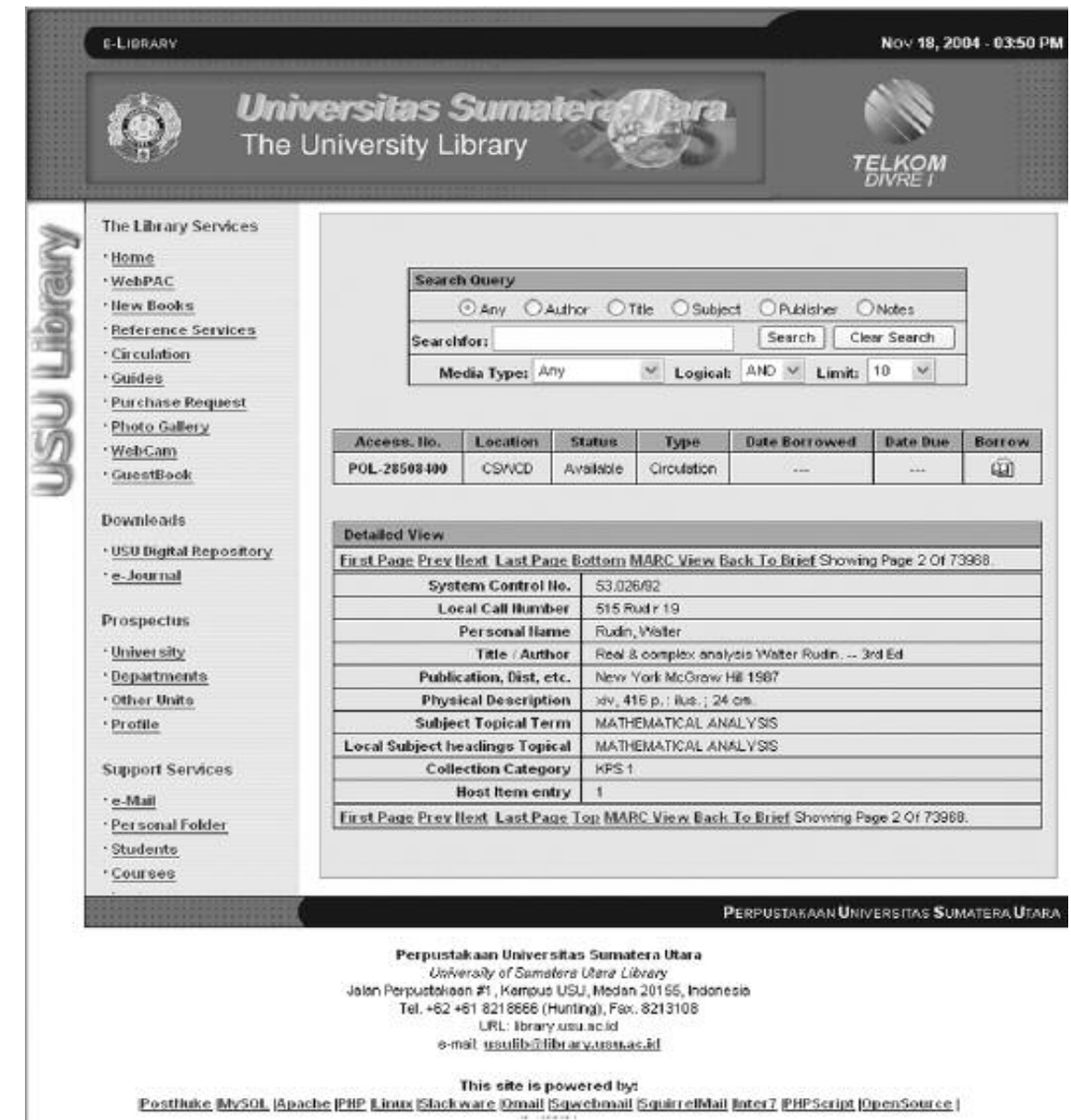

Figura 6. Módulo phpMyLibrary en la University of Sumatera Utara Library 
-Academia e-learning.

http://www.elearningworkshops.com/

-Urbandale public library.

http://www.urbandalelibrary.org/ index.php

-Associació del Professorat

de Tecnologia de Catalunya (UPC).

http://www.aptc.net/aptcnuke/

index.php

- Centro del profesorado de Huelva-Isla Cristina.

http://www.cephuelva.net/cep/ index.php

-Istec: Iberoamerican Science \& Tech Education Consortium. Library. Digital library linkages.

http://dll.istec.org/

\section{Bibliografía}

Cox, Andrew; Yeadon, Jane. «Estrategias prácticas de gestión de contenidos». En: El profesional de la información, 2002, v. 11, n. 6, pp. 466-474.

Hunter, Philip. «The management of content: universities and the electronic publishing revolution». En Ariadne, Junio 2001, n. 28

http://www.ariadne.ac.uk/issue28/cms [revista electrónica]

Pérez Pérez, Carlos. «Manual de referencia rápida para PHP-Nuke».

http://www.forodecanarias.org/doc/nuke/html/ [recurso electrónico]

Portela, Paloma. «Portales, sindicación, contenidos: nuevas oportunidades para los gestores de información». En: El profesional de la información, 2001, v. 10, n. 12, pp. 14-16.

Rodríguez Gairín, Josep Manuel. «Gestión de contenidos con PHP-Nuke». En: Rovira, Cristòfol; Codina, Lluís (dir.). Documentación digital. Barcelona: Sección Científica de Ciencias de la Documentación. Departamento de Ciencias Políticas y Sociales. Universidad Pompeu Fabra, 2003. Isbn 84-88042-39-6.

http://www.documentaciondigital.org

Sennema, Greg. «Creating an internal content management system». En: Computers in libraries, 2004, v. 24, n. 1, pp. 2730

http://www.infotoday.com/cilmag/jan04/ sennema.shtml

Josep Manuel Rodríguez, i Gairín, Departament de Biblioteconomia $i$ Documentació, Universitat de Barcelona, Melcior de Palau 140, 08014

Barcelona.

rodriguez.gairin@ub.edu 